\title{
Real Time Motion Detection using Dynamic Camera
}

\author{
Mayuri Konde \\ Trinity College of Engg. \& \\ Research Pune, \\ India
}

\author{
Manasi Chaudhari \\ Trinity College of Engg. \& \\ Research Pune, \\ India
}

Vidya Kadam

Trinity College of Engg. \& Research Pune,

\author{
Vikas Yadav \\ Trinity College of Engg. \& \\ Research Pune, \\ India
}

\begin{abstract}
Nowadays, security of valuable and secret assets is very important for large organisation companies. Need of an efficient and reliable security system is cause due to limitation of human resources and man power.

High level surveillance system for security is implemented to overcome this limitations and errors. In this system, dynamic camera is used for video surveillance which will feed video stream to system. System will perform various image processing operations to detect the object.
\end{abstract}

\section{Index Terms}

Image processing, voting based motion estimation algorithm, Priority based spatial coding algorithm, content based temporal sampling algorithm, Video Surveillance system.

\section{INTRODUCTION}

Video surveillance systems are a very important in the modern times. Although some people don't like the idea of being watched, surveillance systems improve the public security, allowing the system to detect dangers and the security forces to react in time. Surveillance systems developed in the recent years from simple surveillance systems into complex structures, containing multiple cameras and high end monitoring centers, armed with elegant hardware and software. However, the future of surveillance systems belongs to automatic tools that assist the system operator and notify him on the detection of security threats. It is important, because in complex systems consisting of multiple cameras, the operator cannot notice all the events.

For efficient and reliable surveillance system, high detection rates and low false alarm rates are necessary, both of these ordinary methods most of the time does not work in turbulent territory. To eliminate such difficulties, two different algorithms for camera motion estimation, capturing target frame and object detection. Goal of this paper is to detect objects in motion reliably.

\section{LITERATURE SURVEY}

[1] Feng-Li Lian, Yi-Chun Lin,Chien-Ting Kuo, and Jong-Hann Jean,moving object detection using mobile.

In this method, using moving camera the video is captured. The reference frame is taken first when the camera starts then current frame and the previous frame both are compared. Utilizing the frame differencing technique first the change detection from the captured images can be performed so the binary image can be generated by this technique that can be used to identify the area with significant difference between two frames or from the current frame to the background frame.

[2] D.Wu,Y.T.Hou, Y. Q. Zhang, Real Time transmission of Video

The video data from these cameras should be transmitted in real time to the control room or end-users for further analysing surveillance-related information. However, transmitting real time video over a network is a challenge task because video data usually contain large amount of information quantity and the transmission channel might have limited bandwidth. When the transmission amount of video data exceeds the available bandwidth, excessive video flow in the network might lead to time delay and or packet loss, and further, the real-time performance of video transmission would be degraded.

[3]J. M. Shapiro, B. Andersson, N. Pereira, W. Elmenreich A key solution to video transmission is to reduce the quantity and complexity of original videos but simultaneously preserve the most important message within the original image content. The development of image compression is to reduce the data quantity at the spatial scale for successful transmission. Based on the characteristics of the objects of interest existing in the video images, embedded coding algorithms are well-known techniques for image compression by generating variable bit-rate streams for progression transmission.

\section{BLOCK DIAGRAM}

The following block diagram represents the working of real time motion estimation by using dynamic camera. The diagram is divided into two parts. First one is capturing of frame and second part is comparison and object detection. At first, based quality image frame is captured from data stream of multiple frames using some frame grabbing algorithm. Then that frame is names as a recent or current image. The recent or current image is in RGB format which then converted into grey scale image. After RGB to grey scale conversion Gaussian blur technic is used to reduce noise and sharpness in the image. After above process the recent image is compared with background image. Background is image that if image that captured and updated by camera for every particular time frame for comparison of this two image recent image subtracted from background image after subtraction threshold technique used on resultant image. Blob detection algorithm used on binary image from thresholding, which detect the moving object in that image. After the object detection object is registered and track. 


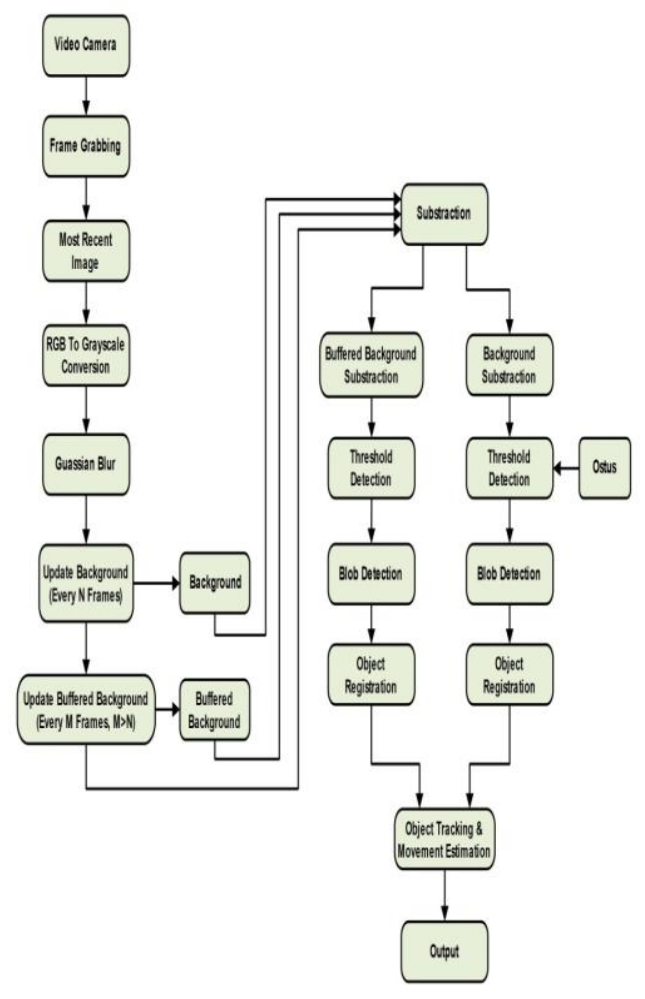

Fig. 1:Block diagram

\section{CAMERA MOTION ESTIMATION}

In voting based mechanism image processing technique is used for motion estimation and edge detection. Image is necessary in tracking, detecting and recognition applications. There are two types' image features Frequency features and amplitude features. Edge information of object is usually use for detect the location of moving object.

Voting base mechanism edge detection of object plays very important role .In dynamic camera surveillance system as camera is always moving, the objects in captured images also look like moving objects, even though they are not. Correct edge information of moving object and still object can be obtained by subtracting to successive frames .Estimation of camera motion is very important for identifying visual information of moving object. Therefore motion of camera should be estimated first using estimated motion of camera output of edge detection is calculated .The morphological erosion dilation are used to get correct and enhance outcome of moving edges.

\section{CONTENT BASED TEMPORAL SAMPLING}

In surveillance system with high end camera devices frame rate is high and change of view cause by motion of dynamic camera is very small due to that consecutive captured images are almost identify .Hence transmitting this identical frames on limited bandwidth is not efficient utilization of bandwidth. Due to this problem the content based temporal sampling method is used.

In content based temporal sampling only one image from $n$ number of consecutive frames is selected. The selected images are selected on basis of content of image .Image containing more information is selected compare to image with less information. Image having more edges having more information. Therefore while selecting images blur images are given least preference over sharp images for frame selection canny edge detection is performed to locate edge pixel then number of are count for changing edges on each frame according to content based temporal sampling algorithm the most important information would be save and less important an identical frame would be removed.

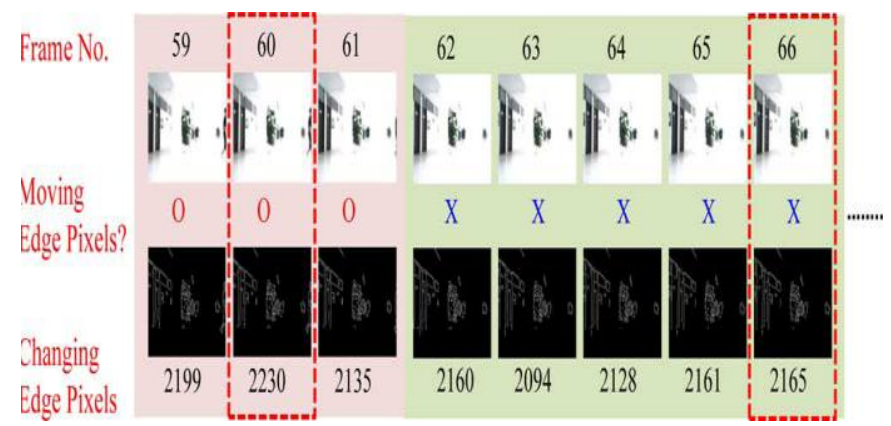

\section{PRIORITY BASED SPATIAL CODING}

Usually, an image frame can be divided into important and unimportant region in spatial domain. The importance can be decided based on the outcome of the moving edge detection. For example, moving objects can be considered as the most important information compared with other static objects and background. Hence, the result of the edge detection can also be used to specify the regions with or without moving objects. Therefore, the spatial coding algorithm can be used to encode the region with important information into a frame of higher visual quality and the region without important information into a frame of lower visual quality. Furthermore, an embedded coding algorithm, such as the set partitioning SPIHT, can be used to progressively encode the visual quality based on the currently determined importance and available bandwidth.

\section{CONCLUSION}

In this paper a smart surveillance system will be implemented which will detect moving object as well as abandoned object with dynamic camera. Motion detection algorithm and various images processing technique will be used for detection of objects in video stream. The surveillance system being implementing is low cost, efficient and highly reliable. This system will give common man access to use sophisticated security system.

\section{REFERENCES}

[1] G. L. Foresti, C. S. Regazzoni, and R. Visvanathan, "Scanning theissue/technology—Special issue on video communications, processingand understanding for third generation surveillance systems," Proc.IEEE, vol. 89, no. 10, pp. 1355-1367, Oct. 2001.

[2] S.Misra,M. Reisslein, and G. Xue, "A survey ofmultimedia streamingin wireless sensor networks," IEEE Communications Surveys \& Tutorials ,Fourth Quarter, vol. 10, no. 4, pp. 18-39, 2008.

[3] Y. Si, J. Mei, and H. Gao, "Novel approaches to improve robustness, accuracy and rapidity of iris recognition systems,” IEEE Trans. IndInf., vol. 8, no. 1, pp. 110-117, 2012.

[4] P. N. Huu, V. Tran-Quang, and T. Miyoshi, "Image compression algorithm considering energy balance on wireless sensor networks, in IEEE Int. Conf. Industrial 
Informatics (INDIN), Osaka, Japan, Jul.13-16, 2010, pp. $1005-1010$.

[5] A. Hampapur,L.Brown, J.Connell,A.Ekin, N. Haas,M. Lu,H.Merkl,S. Pankanti, A. Senior, C.-F. Shu, and Y. L. Tian, "Smart video surveillance: Exploring the concept of multi-scale spatiotemporal tracking, "IEEE Spatial Process. Mag., vol. 22, no. 2, pp. 38-51, Mar. 2005.

[6] D.Wu,Y.T.Hou, and Y. Q. Zhang, "Transporting real-time video over the internet: Challenges and approaches," Proc. IEEE, vol. 88, no. 12,pp. 1855-1877, Dec. 2000.

[7] C. Caione, D. Brunelli, and L. Benini, "Distributed compressive sampling for lifetime optimization in dense wireless sensor networks, "IEEE Trans. Ind. Inf., vol. 8, no. 1, pp. 30-40, 2012.

[8] M. García-Valls, P. Basanta-Val, and I. Estévez-Ayres, "Adaptive real time video transmission over DDS," in IEEE Int. Conf. Industrial Informatics(INDIN), Osaka, Japan, Jul. 13-16, 2010, pp. 130-135.180 IEEE TRANSACTIONS ON INDUSTRIAL INFORMATICS, VOL. 9, NO. 1, FEBRUARY 2013
[9] H. Koto, Y. Hiehata, S. Uemura, and H. Nakamura, "Analysis of control accuracy for access control method based on sample monitoring in interactive tv services," in IEEE Int. Conf. Industrial Informatics(INDIN), Osaka, Japan, Jul. 13-16, 2010, pp. 991-998.

[10] F.-L. Lian, J. K.Yook,D.M. Tilbury, and J. R.Moyne, "Network architecture and communication modules for guaranteeing acceptable control and communication performance for networked multi-agent systems, "IEEE Trans. Ind. Inf., vol. 2, no. 1, pp. 12-24, Feb. 2006.

[11] B. Andersson, N. Pereira,W. Elmenreich, E. Tovar, F. Pacheco, and N.Cruz, "A scalable and efficient approach for obtaining measurements in CAN-based control systems," IEEE Trans. Ind. Inf., vol. 4, no. 2, pp.80-91, May 2008.

[12] J. M. Shapiro, "Embedded image coding using zero trees of wavelet coefficients". IEEE Trans. Signal Process., vol. 41, no. 12 , pp.3445-3462, Dec. 1993. 